\title{
No IV, No Problem: A Cross Sectional Analysis of Antiemetic Therapies in Statewide EMS Protocols
}

\author{
Carlin C. Chuck, NREMT; Thomas J. Martin, BA, NRP; Isabelle H. Moseley, NREMT; \\ Megan L. Ranney, MD, MPH, FACEP; Nicholas Asselin, DO, MS
}

POSTER PRESENTATION ABSTRACT | ORIGINAL RESEARCH CATEGORY

Introduction: Nausea is a common reason for ED presentation and may represent up to 10\% of chief complaints in the prehospital setting. In the collegiate setting, EMS providers often encounter nausea secondary to consumption of alcohol or other drugs. The prehospital management of nausea varies with local protocol and scope of practice. We hypothesized that CBEMS agencies operating at the BLS level may have limited options for antiemetic therapy.

Methods: A cross-sectional analysis of publicly available statewide EMS protocols published within the last ten years was completed in October 2018. The presence of a BLS nausea protocol was defined as the primary outcome of interest; secondary outcomes including the availability of oral (ODT) ondansetron or alternative antiemetic agents were defined a priori. Data were collected independently by two trained reviewers using standardized abstraction forms; inter-rater reliability was assessed using Cohen's kappa ( $\kappa)$ and discrepancies were resolved by the senior author. Descriptive statistics were generated using $\mathrm{R}$ v3.3.2. This study was exempt from IRB review.

Results: 30 model or mandatory statewide EMS protocols were identified using aggregate databases and internet searches; data were abstracted with $\kappa=1$. Of the thirty states, $13.3 \%$ (4/30) have adopted BLS nausea protocols: two allow ODT ondansetron, one allows isopropyl alcohol aromatherapy, and one allows P6 acupressure. No protocols were identified that include intramuscular antiemetics for BLS providers. In addition, 70\% (21/30) of state protocols include ODT ondansetron, but 90.4\% (19/21) of these restrict administration to ALS providers. $46.7 \%$ (14/30) of states include alternative antiemetics for ALS providers such as promethazine and metoclopramide, often as second-line therapies.

Discussion/Conclusions: CBEMS agencies operating at the BLS level are unable to administer ondansetron intravenously or intramuscularly. Though ODT ondansetron and isopropyl alcohol aromatherapy are safe and effective for treatment of nausea in the ED, prehospital adoption remains low. Further research should assess the feasibility, safety, and efficacy of introducing these therapies at the BLS level.

Author Affiliations: From Brown University Emergency Medical Services, Brown University, Providence, RI, USA (C.C.C, T.J.M, I.H.M, N.A.); The Warren Alpert Medical School, Brown University, Providence, RI, USA (T.J.M, M.L.R, N.A.); Program in Emergency Digital Health Innovation, Department of Emergency Medicine, The Warren Alpert Medical School, Brown University, Providence, RI, USA (M.L.R); Division of EMS, Department of Emergency Medicine, The Warren Alpert Medical School, Brown University, Providence, RI, USA (N.A.).

Address for Correspondence: Thomas J. Martin, BA, NRP.

Email: thomas_martin@brown.edu

Conflicts of Interest/Funding Sources: By the JCEMS Submission Declaration Form, all authors are required to disclose all potential conflicts of interest and funding sources. The authors declared that they have no conflicts of interest. The authors declared that they did not receive funding to conduct the program or research associated with this work.

Ethical Compliance: The authors attest that the research associated with this abstract was conducted in accordance with the JCEMS Ethics Guidelines.

Submission History: Received November 30, 2018; accepted for presentation and publication January 22, 2019.

Poster Presentation: This abstract was presented as a poster at the Academic Poster Session of the $26^{\text {th }}$ Annual Conference of the National Collegiate Emergency Medical
Services Foundation; February 23, 2019; Pittsburgh, PA, USA.

Published Online: December 31, 2019

Published in Print: December 31, 2019 (Volume 2: Supplemental 1)

Reviewer Information: In accordance with JCEMS editorial policy, poster presentation abstracts undergo double-blind peer-review by at least two reviewers (JCEMS Editorial Board members and/or independent reviewers) prior to acceptance for presentation and publication. JCEMS thanks the anonymous reviewers who contributed to the review of this work.

Copyright: () 2019 Chuck, Martin, Moseley, Ranney \& Asselin. This is an open access abstract distributed under the terms of the Creative Commons Attribution 4.0 International (CC BY 4.0) License, which permits unrestricted use, distribution, and reproduction in any medium, provided the original author and source are credited. The full license is available at: https://creativecommons.org/licenses/by/4.0/ Electronic Link: https://doi.org/10.30542/JCEMS.2019.02.S1.04 